University of Windsor

Scholarship at UWindsor

2011

\title{
A replenishment control system with uncertain returns and random opportunities for disposal
}

Ben A. Chaouch

University of Windsor

Follow this and additional works at: https://scholar.uwindsor.ca/odettepub

Part of the Business Commons

\section{Recommended Citation}

Chaouch, Ben A.. (2011). A replenishment control system with uncertain returns and random opportunities for disposal. International Journal of Inventory Research, 1 (3), 221-247.

https://scholar.uwindsor.ca/odettepub/20

This Article is brought to you for free and open access by the Odette School of Business at Scholarship at UWindsor. It has been accepted for inclusion in Odette School of Business Publications by an authorized administrator of Scholarship at UWindsor. For more information, please contact scholarship@uwindsor.ca. 


\title{
A replenishment control system with uncertain returns and random opportunities for disposal
}

\author{
Ben A. Chaouch \\ Odette School of Business, \\ University of Windsor, \\ Ontario N9B 3P4 Canada \\ Fax.: (519)-973-7073 \\ E-mail: chaouch@uwindsor.ca
}

\begin{abstract}
We consider a replenishment control system in which product returns play an important role in inventory planning. We focus on the inventory of an individual item that is stored at a single location to meet a constant demand over time. We assume that the total amount of returns accumulated over a period of time can be represented by a compound Poisson process. We further assume that opportunities for inventory disposals or relocation arise occasionally in accordance with a Poisson process. We not only seek to resolve the issues of when to order and how much to order, we also consider the question of when to dispose of excess inventory and by how much. Inventory reductions occur when the opportunity for a disposal arises and the inventory position is deemed too high. After each disposal the inventory position is restored to a specified base-stock level. We develop a cost model of this system and highlight its properties through an extensive numerical study.
\end{abstract}

Keywords: inventory management; product returns; disposals; stochastic models; level-crossing theory.

Reference to this paper should be made as follows: Chaouch, B.A. (xxxx) 'A replenishment control system with uncertain returns and random opportunities for disposal', Int. J. Inventory Research, Vol. X, No. Y, pp.000-000.

Biographical notes: Ben A. Chaouch is an Associate Professor of Management Science at the Odette School of Business, University of Windsor, Canada. He teaches courses in operations research, quality management and control, and operations management. He received a Masters degree in Operations Research from Stanford University, and a $\mathrm{PhD}$ in Management Sciences from the University of Waterloo. His work has appeared in Management Science, Naval Research Logistics, Production and Operations Management, European Journal of Operational Research, and other publications.

\section{Introduction}

Customers return products to vendors for many different reasons. One common reason is when a customer returns non-conforming batches because the product's quality or performance is not to her satisfaction. Stock et al. (2002) cite the following reasons for the expanding volume of product returns observed in North America: the growing 
popularity of electronic retailing, the convenience of catalogue purchasing, longer warranty periods, and the low tolerance of customers for imperfect products.

In the manufacturing sector, new environmental laws and take-back regulations have forced manufacturers to give due consideration to the management of return flows of products that may require remanufacturing, refurbishing, recycling, reuse or disposal (see Fleischmann et al., 1997; Blackburn et al., 2004). Current industry practice is to have all returns shipped to a central facility where they are evaluated and reused accordingly.

The management of product returns has also become a top priority in retailing with the proliferation of easy and flexible returns policies at many levels in the supply chain. In order to please customers and make the return process a competitive advantage, free return policies are now a standard feature offered by many online retailers such as Zappos.com, the web's popular shoe store (http://www.zappos.com). Zappos makes the process of returning unwanted shoes easy and convenient. Customers are given the opportunity to easily download a pre-paid return shipping label if they do not like the shoe and want to ship it back. While customers appreciate the convenience of free returns, one estimate pegs the shipping costs Zappos incurs for this service at around $\$ 100$ million.

Vendors also use generous returns policies as a way to encourage customers to stock and price products aggressively in order to increase sales. Such is the case for many high-volume and high-value products in the heavy equipment, appliance, and computer industries, where inventory return requests are accepted at no cost to the customer (Lonn and Stuart, 2003). Lonn and Stuart relate Caterpillar's experience with returns and mention that "of the 36.4 million line items that are shipped annually from the company's global distribution center, hundreds of thousands of unsold and unused items are eventually requested for returns by dealers". Caterpillar needed a formula that can help with the decision of whether to accept or deny a return.

These examples point to some important issues of inventory management for vendors that must deal with large quantities of returned products. Fleischmann et al. (1997) and Blackburn et al. (2004) discuss these issues at length in the context of reverse supply chains for commercial products. One important question of inventory management raised in these papers is: How should the vendor choose appropriate inventory policies when the flow of returns in quantity, quality, and timing cannot be predicted precisely in advance? In most cases, product returns exacerbate the inventory control problem. The reason is that, while inventory is being depleted by demand, the inventory position between regular replenishments may increase to unwanted levels because of frequent large returns. Once this inventory gets too large, the vendor may find it necessary or economical to offload the excess through disposal or other means in order to keep the inventory holding costs down.

Many researchers have addressed this general problem in different ways and have proposed different quantitative models that can be used to determine optimal replenishment and disposal policies. In Section 2, we discuss the extensive literature that has addressed the problem of product returns and their impact on inventory management. In Section 3 we give a detailed statement of the inventory system with stochastic returns and disposals we study in this paper. Along the way, we relate our work to this literature and show how our approach differs from those taken in previous studies. Section 4 presents a detailed analysis of the model, and Section 5 gives our conclusions. 


\section{Literature review}

The literature on inventory models that incorporates product returns and disposals has a long history going back to the 1960s. This literature includes both deterministic and stochastic models. For representative deterministic models, see Schrady (1967), Richter (1996), Teunter (2001), and Teunter and Van der Laan (2002) for EOQ-like models and Beltrán and Krass (2002) for a multi-period dynamic model.

The literature on stochastic models can be divided into two important streams. One stream of models assumes that an item when returned is not quite ready for use and may require repair or remanufacturing before it can be added to the inventory available for sale or the so-called serviceable inventory. So each returned item is considered to be in a state of repair and a decision will follow as to whether it should be repaired or remanufactured and then added to serviceable inventory or discarded for good. These models make a clear distinction between serviceable inventory and repairable inventory and the two stockpiles are kept separate. The literature refers to this dichotomy as the two-echelon classification (Fleischmann et al., 1997). The general problem is to manage the two inventories concurrently. The goal is to determine the optimal quantities of new items to purchase and the amount to repair or to remainder of the repairable inventory, given a random demand for the product and random returns.

This stream of models can further be divided according to the approach used to formulate the problem. Simpson (1978), Inderfurth (1997), Mahadevan et al. (2003), and Karaer and Lee (2007) all use a periodic review approach. In Simpson's model, the inventory manager, who wants to satisfy a random demand for serviceables in an n-period problem with random returns, must decide how many new items to purchase and how many units to repair and/or junk of the repairable stockpile in each period given the current quantities of serviceables and repairables. Under the assumption of zero lead times for procurement and repair, he proves the optimality of a policy that is specified by three critical levels: a repair-up-to-level, a purchase-up-to-level, and a scrap-down-tolevel. Inderfurth (1997) shows that this form of policy remains optimal when fixed replenishment and repair lead times are added to Simpson's model.

Mahadevan et al. (2003) use a periodic review policy in a continuous-time setting. At each review period the manufacturing facility makes an order to raise the inventory position of serviceables to some fixed replenishment level S. The order is a two-step process. First the returned, but not yet reworked, items (or carcasses) are released for remanufacture to raise the inventory position to $\mathrm{S}$. If the present inventory of carcasses is not enough for the inventory position to reach $\mathrm{S}$, the shortfall is made up by placing an order for brand new items, which takes longer to manufacture. Demands and returns occur according to two independent Poisson processes with different intensities. Karaer and Lee (2007) quantify the benefit of obtaining visibility and advance information on return flows (i.e., returns currently being evaluated, reworked, and in-transit to DC stockpile) when setting optimal base-stock inventory policies.

Of the models that use a continuous review approach, Muckstadt and Isaac (1981) is one of the primary early works to use a reorder-point order-quantity model formulation of the problem. The location has a facility to repair or refurbish returned items and a different facility to store serviceable inventory. A returned item joins a queue at the repair facility and waits its turn to be repaired. Once an item is repaired it is quickly added to the inventory waiting for sale. The processes generating demands and returns are assumed to be Poisson with different means. The authors approximate the stationary 
distribution for net inventory and use it to develop a cost rate function. Van der Laan et al. (1996a, 1996b) extend Muckstadt and Isaac's framework to include inventory disposals when the total number of returns reaches a critical level at the repair facility. They propose different approximation schemes, which are used to derive the average cost per time.

Van der Laan et al. (1999) also use a continuous-review model approach. In this model, serviceable inventory is also supplied from two sources, remanufactured returns and newly manufactured products. Both returned and new products take a fixed lead time to manufacture. The authors analyse the impact of PUSH and PULL control strategies on costs for some given replenishment policies.

The other important stream of models focuses primarily on serviceable inventory and its up-and-down fluctuations over time. Demands cause inventory to decrease, while product returns (already repaired or restocked as-is) or new order receipts cause it to increase. Repair or remanufacturing activities and the delays they engender are not considered explicitly as in the first category of models. In this group of models we also find periodic-review vs. continuous-review formulations. Cohen et al. (1980) consider the situation in which a constant fraction of the inventory sold in each period will be returned in good condition to the original stockpile after a fixed number of periods. The model allows for a fixed fraction of on-hand inventory to be lost to decay each period. The authors show that a myopic base-stock policy is optimal when the stock issued to satisfy demand is returned after one period.

Products are often sold in returnable containers (e.g., pallets). Kelle and Silver (1989) consider the inventory of containers that are sent outside the firm to satisfy customers' needs. A container may take a random length of time before it can be retrieved and reused again. There is also a probability for a container to go missing and never make it back. So orders for new containers are also required. A purchasing policy of these containers is determined in a discrete-time model so as to minimise total expected costs under a prescribed service level.

Fleischmann and Kuik (2003) consider a standard single-item dynamic inventory model with i.i.d. 'demands'. Here the demand in each period can be positive or negative and is understood as the difference between the amount sold and the amount returned. A negative demand results in an inventory increase. When an $(s, S)$ ordering policy is used and inventory disposals are not considered, the authors show that the model can be transformed into a regular stochastic dynamic inventory model with no returns.

The majority of papers described thus far assume that the process generating returns is independent of the demand process. This is often true when the item in question is sold in large quantities to a large base of customers and returns policies are sufficiently flexible. However, for rented or leased products where a service contract of fixed length is generally in place, the dependence between demands and returns is somewhat strong. Kiesmuller and Van der Laan (2001) study an inventory model in a finite planning horizon that allows for the return process to depend explicitly on the demand stream. Each sold item, if returned, is retrieved after a fixed length of time. The decision of whether to remanufacture or scrap a returned item is done at retrieval time.

Heyman (1977), Fleischmann et al. (2002), and Yuan and Cheung (1998) use a continuous-review approach. These authors also restrict attention to serviceable inventory and its random behaviour over time. Heyman (1977) examines a single-item inventory system in which the stock level goes up and down as items are returned and demands are satisfied, respectively. The inventory level is allowed to fluctuate between two given 
limits. When inventory reaches the lower limit, the arriving demands are satisfied on an emergency basis at no extra cost (i.e., backordering or lost sales are not allowed). When inventory reaches the upper limit, a returning item is automatically disposed of. The objective is to find the inventory upper-limit at which disposals are to take place.

Fleischmann et al. (2002) analyse an inventory model with stochastic item returns. Replenishment orders are performed according to an (s, Q) policy based on the inventory position. They develop an expression for the long-run average costs when the returns and demands form independent Poisson processes. Yuan and Cheung (1998) address the situation of leased items that customers use for a random length of time and may or may not return for reasons outside the control of the firm. In other words, the return stream is entirely dependent on the demand stream, which is assumed to be Poisson. There is a fixed probability that the leased item will not be returned. Replenishment decisions are made according to an (s, S) policy.

Recently there has also been research that extends the two-echelon structure mentioned above to more general structures. For example, DeCroix and Zipkin (2005), DeCroix et al. (2005), and DeCroix (2006) study multi-stage inventory systems that consider the impact of product returns on echelon inventories. In these systems, returned components or subassemblies are forwarded to their appropriate stage simultaneously.

One of the assumptions inherent in most of the models described above relates to the way in which the return process is often represented. Returns are often assumed to arrive one at a time in a random fashion. The model we are about to present considers the next natural form of this process. That is, returns arrive in batches of varying sizes at random times; an important situation often faced by both manufacturers and retailers. Furthermore, our model includes the possibility of disposing or reallocating excess inventory when such a need arises in a manner that has not been considered before.

\section{Model description}

We focus on the inventory of an individual item that is stored at a single location, e.g., a distribution centre (DC). The inventory of this item is continuously monitored as it is depleted by demand. It is replenished by placing orders whenever the inventory position reaches a reorder point. We assume that the demand for this item is deterministic and occurs at a known constant rate of D units per unit time. Fisher (1997) characterises products as functional, products that have low-demand uncertainty and long-life cycle or innovative, products that have high-demand uncertainty and short-life cycle. Our model applies to the first category of products or those products that are in the mature stage of the life cycle. The inclusion of a random demand would certainly lead to a richer model; unfortunately, analysis of the model taken as a whole quickly becomes unwieldy. We believe that insight will not be diminished by our restriction to a uniform demand rate. It is well-established in the literature that general EOQ models are fairly robust; even large deviations in parameter values produce only small cost errors (Silver et al., 1998). The present model inherits this property as well.

On occasions, however, the inventory of this item may experience upward jumps in-between normal replenishments because a quantity of this item has been returned and re-integrated into the stockpile. We assume that returns occur at random points in time and the quantity returned is a random variable. This is often the situation witnessed in practice as many returns arrive at the $\mathrm{DC}$ from varying sources and in varying quantities 
and, thus, their arrival times and sizes are not known in advance. In what follows, we assume that the return process can be represented by a compound Poisson process denoted by $R(t) . R(t)$ represents the total number of units returned to serviceable inventory by time $t$. That is, $R(t)$ is given by $R(t)=\sum_{i=1}^{N(t)} R_{i}$, where $\{N(t), t \geq 0\}$ is a Poisson process with rate $\lambda$, and $R_{i}$ is the amount returned at the epoch of the $i^{\text {th }}$ event of $N$. $\left\{R_{i}, i=1,2, \ldots\right\}$ is a sequence of i.i.d. random variables with a common mean $E[R]$ and variance $\operatorname{Var}[R] . N(t)$ and $\left\{R_{i}\right\}$ are assumed to be independent. Moreover, we make the assumption that $\lambda E[R]<D$, i.e., the average number of units returned per unit time is less than the demand rate. Otherwise, the product is liable to be discontinued.

Our model can be viewed to belong to the second category of models described above that concentrates mainly on serviceable inventory and its day-to-day increases and decreases. The majority of models that use a continuous review procurement policy assume that the return process is a simple Poisson process, a reasonable approximation for some products. But, for many other products, returns can be larger than unit-size. Our model is intended to fill this gap. We will be able to assess the main effects of batched returns on the optimal replenishment and disposal policy. We note that the return process need not be independent of the demand rate $D$. Our results will remain unaffected if, for instance, the frequency or magnitude of returns were to depend explicitly on $D$.

We further assume that the item in question is managed according to the following inventory policy. Whenever the inventory position drops to the order point s or lower, we order up to the target level $s+q$. That is, an order for $q$ units is placed. Orders take a fixed lead time $L$ to arrive. Moreover, due to the uncertain returns that are eventually restocked for sale, the inventory position may rise to unacceptable levels. When this happens and the inventory position is greater than $s+q+Q$, an inventory reduction is considered in order to re-establish current levels back to some prescribed level $s+q+M$ for $0 \leq M \leq Q$ through disposal or reallocation of the excess. Thus, an action to bring the inventory position down to $s+q+M$ is taken only when a surplus inventory of $Q$ units has built up over and above the target level $s+q$.

Our use of the word disposal here does not necessarily mean that the DC will turn to the first available liquidator or discount chain to quickly get rid of its excess inventory. Rather, we mean that the DC is on the lookout for the best opportunity that may come along to make good on its extra inventory. For example, disposal could mean finding a secondary market for the surplus inventory or redistributing the excess from one location to another location with demand for it. In general, opportunities of this kind arise in an unpredictable fashion and, thus, will exhibit some variability. Let us say that the product is carried in inventory at multiple locations and one or more of these locations find themselves in a backorder or low-inventory position. If the current location happens to be overstocked at those opportune times, the excess can be deployed quickly to the needy locations. Similarly, the price of the product in the secondary (e.g., a spot) market has turned favourable again and it makes sense for the location to take advantage of this opportunity to dispose of its excess and so on. Obviously, events of this type cannot be foretold and, thus, can be considered to happen at random times. For ease of presentation and tractability reasons, we assume that the times at which such opportunities materialise occur according to a Poisson process with rate $\theta$, which is independent of $N(t)$.

Almost all models that consider disposals in the context we describe assume that disposition of returns occurs at the receiving stage of the process (Van der Laan et al., 
1996a, 1996b; Kiesmuller and Van der Laan, 2001; Karaer and Lee, 2007; Blackburn et al., 2004). That is, the condition of product returns is assessed as early as possible and a disposition decision is carried out soon enough if the return is found to have no recoverable value. Van der Laan et al. (1996b) include a disposal option at the repair stage, where incoming returns are automatically discarded once the repairable inventory has reached a given limit. However, disposals can and should also occur at the serviceable inventory level if too many and frequent returns leave in their wake too much inventory. Van der Laan et al. (1996b) remark that a policy, which bases disposals on the overall inventory position, is often economically more attractive. In the proposed model, unlike the studies just described, disposals depend on the current state of the inventory position for serviceables. Thus, in terms of the previous work, our model can be viewed as an extension of the models presented in Heyman (1977), Muckstadt and Isaac (1981), Fleischmann et al. (2002), van der Laan et al. (1996a) and (1996b). These papers, however, consider different demand or return processes or, in most cases, do not include a disposal option.

We develop a cost model of this system and present a solution procedure for determining the optimal policy parameters. Our objective is to minimise the expected system cost per unit time, which is the summation of the procurement cost, holding cost, shortage cost and disposal cost. We assume that unsatisfied demand is not lost, but can be backordered.

The ordering and disposal policy considered in this paper, specified by the four control parameters $s, q, M$, and $Q$, is not only intuitive and easy to implement; it may also represent the optimal policy for the problem at hand based on our computational experience. While it is not easy to establish its optimality in the general context of our model, a policy structure close to it has been proven to be optimal under a different set of model assumptions (see Simpson, 1978 and Inderfurth, 1997). Our goal is to evaluate all the relevant costs associated with the inventory process for any policy in this class. The next section presents our model analysis.

\section{Model analysis}

To present our solution methodology, we need to introduce the following random variables to describe the behaviour of the system over time. For each time $t \geq 0$, let

$I(t)=$ inventory on hand

$B(t)=$ backorders outstanding

$I N(t)=$ net inventory $=I(t)-B(t)$

$I O(t)=$ inventory on order

$I P(t)=$ inventory position $=I N(t)+I O(t)$

$D(t, t+L)=$ demand during lead time $L$

$R(t, t+L)=$ total number of units returned to serviceable inventory during lead time $L$

$S(t, t+L)=$ total number of units disposed of (or withdrawn) from inventory during lead time $L$. 
A typical sample path of the inventory process is shown in Figure 1.

Figure 1 A typical sample path of the inventory process (see online version for colours)

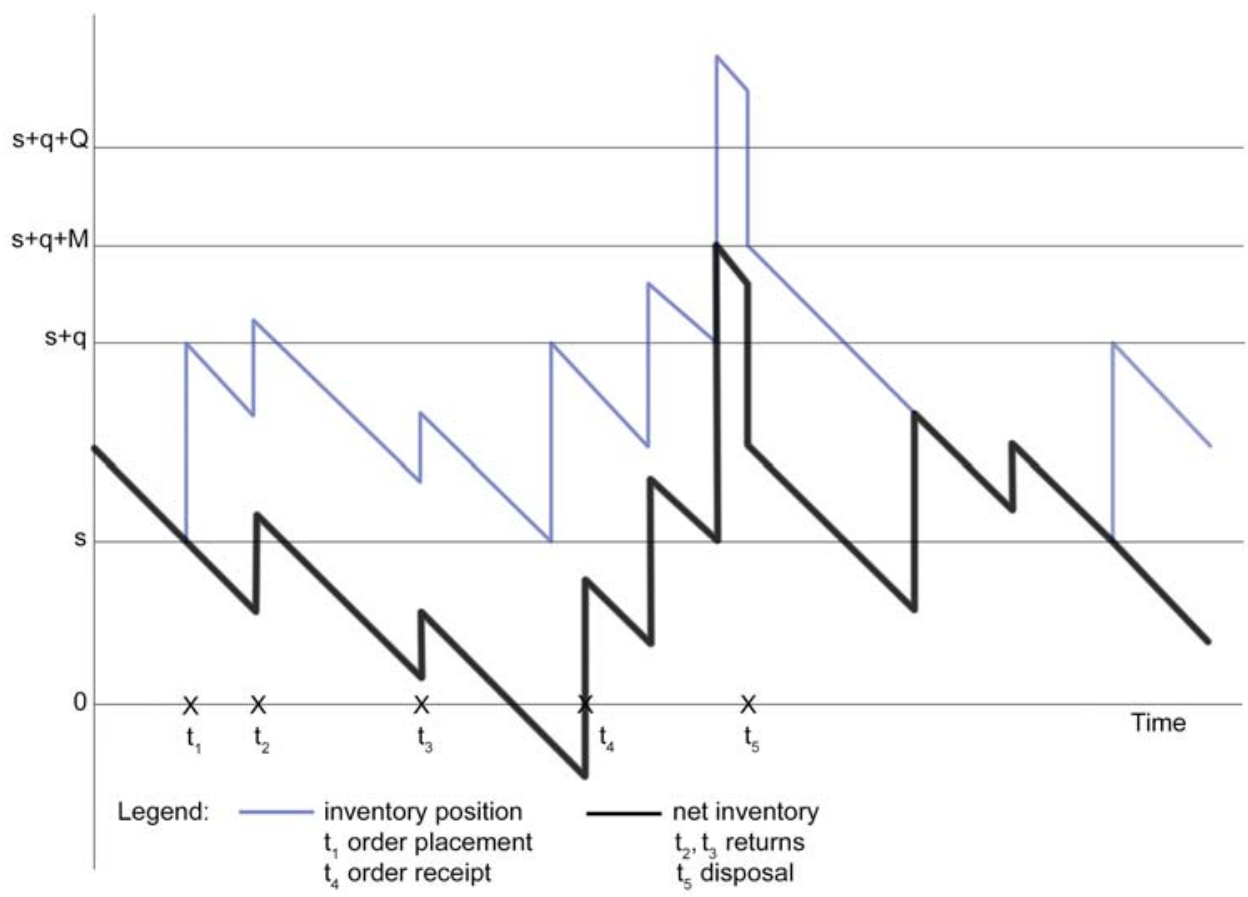

The general method of attack that is commonly used in the literature to derive the performance measures of this type of system is to follow a four-step approach as outlined in Zipkin (2000, p.187). These steps are:

a determine the limiting distribution of $I P(t)$

b describe the relationship between $I N(t), I P(t), D(t, t+L), R(t, t+L)$, and $S(t, t+L)$

c determine (or use a reasonable approximation for) the limiting distribution of $I N(t)$

d derive formulas for the relevant cost measures.

Our analysis begins with the derivation of the cost rate function under the assumption that the replenishment lead time $L$ is small and can be neglected. In this case $s$ can be reduced to 0 with the understanding that stockouts are not to occur. In a later section we consider the case where the lead time of a replenishment order is a positive constant $L$.

\subsection{Cost model with zero lead time}

In this case the inventory position, which is also the inventory on hand, will be denoted by $I P_{0}(t)$. Our first preoccupation is to derive the steady-state distribution of $I P_{0}(t)$. Once this is done it will be a relatively simple exercise to obtain the relevant cost expression in terms of the policy parameters $q, M$ and $Q$. Toward this end, let $F(x)=\lim _{t \rightarrow \infty} P\left(I P_{0}(t) \leq x\right)$ represent the stationary probability distribution function of the 
inventory position. We assume that the parameters of the model are such that the limit exists and is well defined. Also let $f(x)=d F(x) / d x$, where the derivative exists, be the stationary density of the inventory level at $\mathrm{x}$. We shall use a level-crossing approach to find a closed-form expression for $f(x)$. Although the level-crossing technique (LCT) was originally developed to find waiting time distributions for certain queueing models, it has evolved into a versatile method for obtaining probability distributions in many other stochastic models (see, for example, Brill, 2008; Azoury and Brill, 1986, 1992; Brill and Chaouch, 1995; Chaouch, 2001, 2007). The method will allow us to determine detailed rate balance equations from which $f(x)$ can be extracted. For theoretical underpinnings of the method, we refer the reader to Brill (2008) and Brill and Posner $(1977,1981)$.

The basic principle behind LCT is that in order to derive $f(x)$, we examine crossing rates of the $I P_{0}(t)$ process into and out of some well-chosen state intervals. That is, for each level $x \in[0,+\infty)$, we establish a flow rate equation so that the long-run total down-crossing rate equals the long-run total up-crossing rate (for example, see Brill, 2008). This flow balance analysis equating the total flow into a state to the total flow out of the state has its roots in Markov-chain studies.

Let us divide the state space $[0,+\infty)$ into four non-overlapping intervals: $[0, q)$, $[q, q+M),[q+M, q+Q)$, and $[q+Q,+\infty)$. Also let $f_{1}(x), f_{2}(x), f_{3}(x)$ and $f_{4}(x)$ refer to $f(x)$ in these four intervals, respectively. A direct application of LCT yields the following system of rate balance equations:

$$
\begin{gathered}
D f_{1}(x)+\lambda \int_{0}^{q} \bar{G}(q-y) f_{1}(y) d y=\lambda \int_{0}^{x} \bar{G}(x-y) f_{1}(y) d y+D f_{1}(0)+D f_{2}(q) \\
0 \leq x<q \\
D f_{2}(x)+\lambda \int_{x}^{q+M} \bar{G}(q+M-y) f_{2}(y) d y=\lambda \int_{q}^{x}[\bar{G}(x-y)-\bar{G}(q+M-y)] f_{2}(y) d y \\
+\lambda \int_{0}^{q}[\bar{G}(x-y)-\bar{G}(q+M-y)] f_{1}(y) d y \\
+\theta \int_{q+Q}^{+\infty} f_{4}(y) d y+D f_{3}(q+M) \\
q \leq x<q+M \\
D f_{3}(x)+\lambda \int_{x}^{q+Q} \bar{G}(q+Q-y) f_{3}(y) d y=\lambda \int_{q+M}^{x}[\bar{G}(x-y)-\bar{G}(q+Q-y)] f_{3}(y) d y \\
+\lambda \int_{q}^{q+M}[\bar{G}(x-y)-\bar{G}(q+Q-y)] f_{2}(y) d y \\
+\lambda \int_{0}^{q}[\bar{G}(x-y)-\bar{G}(q+Q-y)] \\
f_{1}(y) d y+D f_{4}(q+Q) \\
q+M \leq x<q+Q
\end{gathered}
$$




$$
\begin{gathered}
D f_{4}(x)+\theta \int_{x}^{+\infty} f_{4}(y) d y=\lambda \int_{q+Q}^{x} \bar{G}(x-y) f_{4}(y) d y+\lambda \int_{q+M}^{q+Q} \bar{G}(x-y) f_{3}(y) d y \\
+\lambda \int_{q}^{q+M} \bar{G}(x-y) f_{2}(y) d y+\lambda \int_{0}^{q} \bar{G}(x-y) f_{1}(y) d y \\
q+Q \leq x<+\infty
\end{gathered}
$$

The normalising condition is given by

$$
\int_{0}^{q} f_{1}(x) d x+\int_{q}^{q+M} f_{2}(x) d x+\int_{q+M}^{q+Q} f_{3}(x) d x+\int_{q+Q}^{+\infty} f_{4}(x) d x=1 .
$$

where $G(x)$ is the cumulative distribution of the random variable $R_{i}$, the amount added to serviceable inventory when a return occurs, and $\bar{G}(x)=1-G(x)$.

The validity of the steady-state flow-rate equations given in (1) to (5) stems from our assumption that both returns and disposal opportunities are generated by Poisson streams. Brill and Posner (1977, 1981) and Brill (2008) have supplied a general set of conditions under which rate balance equations of this type hold when steady-state conditions exist.

Equation (2), for example, can be given the following intuitive explanation. For each $x \varepsilon[q, q+M)$, consider the inventory process $I P_{0}(t)$ and its sample-path exits and entrances of the set of states $[x, q+M)$. The left-hand side of (2) represents the long-run expected rate at which $I P_{0}(t)$ exits $[x, q+M)$, while the right-hand side represents the long-run expected entrance rate of this same set. The exit rate of the set $[x, q+M)$ consists of two parts. These are the two terms on the left-hand side of (2). For the first part, for small $d x$, we can interpret $f_{2}(x) d x$ as the long-run probability that $I P_{0}(t)$ is in the interval $(x, x+d x)$. The average demand rate is $\mathrm{D}$ units per unit time and so a continuous down-crossing of level x (or exit) occurs at the long-run rate of $D f_{2}(x)$. This result follows from the basic theorem of level crossing theory (see Brill and Posner, 1981; Brill, 2008). The other part of the exit rate is when $I P_{0}(t)$ is at some level y between $\mathrm{x}$ and $q+M$ with probability $f_{2}(y) d y$ and a return of more than $q+M-y$ units occurs at that moment with probability $\bar{G}(q+M-y)$, which leads to an instantaneous jump out of interval $[x, q+M)$. The integral gives the total probability of this event and when multiplied by $\lambda$, the average return rate per unit time, we get this part's contribution to the exit rate.

Now consider the entrance rate of the set of states $[x, q+M)$. The total rate is the sum of four distinct parts. These are the four terms shown on the right-hand side of (2). The first two terms represent the rate at which returns, that find $I P_{0}(t)$ at some level y either between $q$ and $x$ (state 2) or between 0 and $q$ (state 1), will raise inventory by at least $x-y$ units, but no higher than $q+M-y$ units. The third term represents the rate at which inventory reductions are performed because $I P_{0}(t)$ happens to be in excess of $q+Q$ units and an opportunity for a disposal or withdrawal has presented itself. Such an action results in an instantaneous entrance into the set $[x, q+M)$. This follows from our stated policy that disposals restore inventory back to stock level $q+M$. Finally, the fourth term $D f_{3}(q+M)$ is the continuous down-crossings (or entry) rate into $[x, q+M)$, which occurs when $I P_{0}(t)$ is in the neighbourhood of $q+M$.

Then, by equating the total exit rate and the total entrance rate of the set of states $[x, q+M)$, we arrive at the rate balance equation given in (2) for each $x$ in $[q, q+M)$. Equations (1), (3) and (4) are obtained by similar reasoning. 
Our next task is to find a solution to the system of equations given in (1) to (5). To achieve this, we need to consider a specific distribution for $G(x)$. In what follows, we confine our analysis to the case where the amount returned $R_{i}$ is exponentially distributed with parameter $\mu$ in order to develop useful insights into the effect of batched returns on procurement and disposal policies. This assumption is appropriate for returned products since, generally, returns are expected to be just smaller amounts of a certain product, not entire pallets or cases. For an exponential random variable a large portion of the amount returned will be below the mean. The other reason for this choice is that this distribution is somewhat easier to analyse than any other. Other forms of distribution can also be considered; however, the computational requirements become arduous and long without providing additional insight. Thus, specialising to a distribution for the amount returned given by $\bar{G}(x)=e^{-\mu x}, x \geq 0$, we solve the system of equations (1) to (5) to find that $f(x)$ can be given by

$$
\begin{array}{ll}
f_{1}(x)=\frac{1-\alpha e^{-a \mu x}}{A(q, M, Q)}, & 0 \leq x<q \\
f_{2}(x)=\frac{\alpha\left[1-e^{-a \mu q}\right] e^{-a \mu(x-q)}}{A(q, M, Q)}, & q \leq x<q+M \\
f_{3}(x)=\frac{r+a-\alpha r e^{-a \mu(x-q-Q)}}{\bar{A}(q, M, Q)}, & \\
f_{4}(x)=\frac{a(r+1) e^{r \mu(x-q-Q)}}{\bar{A}(q, M, Q)}, & q+Q \leq x<+\infty
\end{array}
$$

where

$$
\begin{aligned}
& \alpha=\lambda / \mu D, a=1-\alpha, \eta=\theta / \mu D, \\
& r=\left[\eta-a-\sqrt{(\eta-a)^{2}+4 \eta}\right] / 2,
\end{aligned}
$$

and

$$
\begin{aligned}
& A(q, M, Q)=q+\frac{(r+a)\left[1-e^{-a \mu q}\right][Q-M-1 / \mu r]}{\left[(r+a) e^{a \mu M}-r e^{a \mu Q}\right]}, \\
& \bar{A}(q, M, Q)=\frac{\left[(r+a) e^{a \mu M}-r e^{a \mu Q}\right] A}{\left[1-e^{-a \mu q}\right]} .
\end{aligned}
$$

This expression for $f(x)$ can be found directly by turning equations (1) to (4) into a set of four ordinary differential equations (ODE) and then employing the relevant techniques of ODE together with the normalising condition (5) to arrive at the solution shown in (6). We shall not go into the details of the derivation since, while lengthy, it is fairly straightforward.

We are now in a position to derive the total of the expected cost of procurement, the cost of holding stock, and the cost of disposal per unit time. Let us denote by $I P_{0}$ the random variable whose probability density function is given by (6). $I P_{0}$ represents the 
long-run inventory position (and also on hand in this case). To present an expression of the cost rate function, let us define

$K_{1}=$ fixed cost of a replenishment order

$C_{1}=$ procurement cost per unit

$K_{2}=$ fixed cost of disposal

$C_{2}=$ disposal cost per unit

$h=$ inventory carrying cost per unit per unit time

$J(q, M, Q)=$ expected total costs per unit time.

The total cost $J(q, M, Q)$ can be given as follows:

$$
\begin{aligned}
J(q, M, Q)=h E\left[I P_{0}\right] & +\left(K_{1}+C_{1} q\right) D f_{1}(0) \\
& +\theta \int_{q+Q}^{+\infty}\left[K_{2}+C_{2}(x-q-M)\right] f_{4}(x) d x,
\end{aligned}
$$

where

$$
E\left[I P_{0}\right]=\int_{0}^{q} x f_{1}(x) d x+\int_{q}^{q+M} x f_{2}(x) d x+\int_{q+M}^{q+Q} x f_{3}(x) d x+\int_{q+Q}^{+\infty} x f_{4}(x) d x,
$$

$D f_{1}(0)$ is the expected number of orders per unit time, and the last term in the summation is the expected disposal costs per unit time.

In general product returns go through some form of handling or refurbishing before they become resalable. Thus, additional costs are involved in the process. Suppose that the cost of refurbishing a returned product is fixed and is independent of the time it took to spruce up the unit. Let this cost be $C_{3}$ per unit. In this case, since the average number of returned units per unit time is $\lambda / \mu$ in our model, the expected total refurbishment cost per unit time is simply $C_{3}(\lambda / \mu)$. Hence this cost component is a constant in our context and, thus, can be omitted from the objective function.

In situations where $C_{3}$ depends on time and is expressed in $\$ /$ unit/unit-time then, in order to evaluate the expected total refurbishment costs per unit time, one needs to know the average refurbished inventory at any point in time. Unfortunately, this quantity is not easy to calculate exactly. Nonetheless, in Section 4.2, we propose a heuristic approach to approximate this cost component.

Next, using (6) and carrying out the operations, it can be shown that

$$
E\left[I P_{0}\right]=\frac{q^{2} / 2+a_{1} q}{A}+\frac{a_{2}-a_{3} q-a_{4} M+a_{5} Q+a_{6}(2 q+Q+M)(Q-M)}{\bar{A}},
$$

where the constants $a_{1}$ to $a_{6}$ above are given by

$$
\begin{aligned}
& a_{1}=\frac{\alpha}{a \mu}, \quad a_{2}=\frac{a}{\mu^{2}}\left[\frac{r+1}{r^{2}}-\frac{\alpha}{a^{2}}\right], \quad a_{3}=\frac{1}{\mu}\left[\alpha+\frac{a(r+1)}{r}\right], \\
& a_{4}=\frac{\alpha}{\mu}\left[1+\frac{r}{a}\right], \quad a_{5}=\frac{1}{\mu}\left[\frac{\alpha r}{a}-\frac{a(r+1)}{r}\right], \quad a_{6}=\frac{r+a}{2} .
\end{aligned}
$$

Moreover, $D f_{1}(0)=a D / A$, and the last term given as an integral in (7) is given by 


$$
\frac{\theta a(r+1)}{\mu r \bar{A}}\left[C_{2}\left(\frac{1}{\mu r}+M-Q\right)-K_{2}\right]
$$

Figure 2 Cross sections of the cost rate function $J(q, \delta, Q)$ evaluated at an optimal point $(q, \delta, Q)=(33.98,0.76,162)$ (see online version for colours)
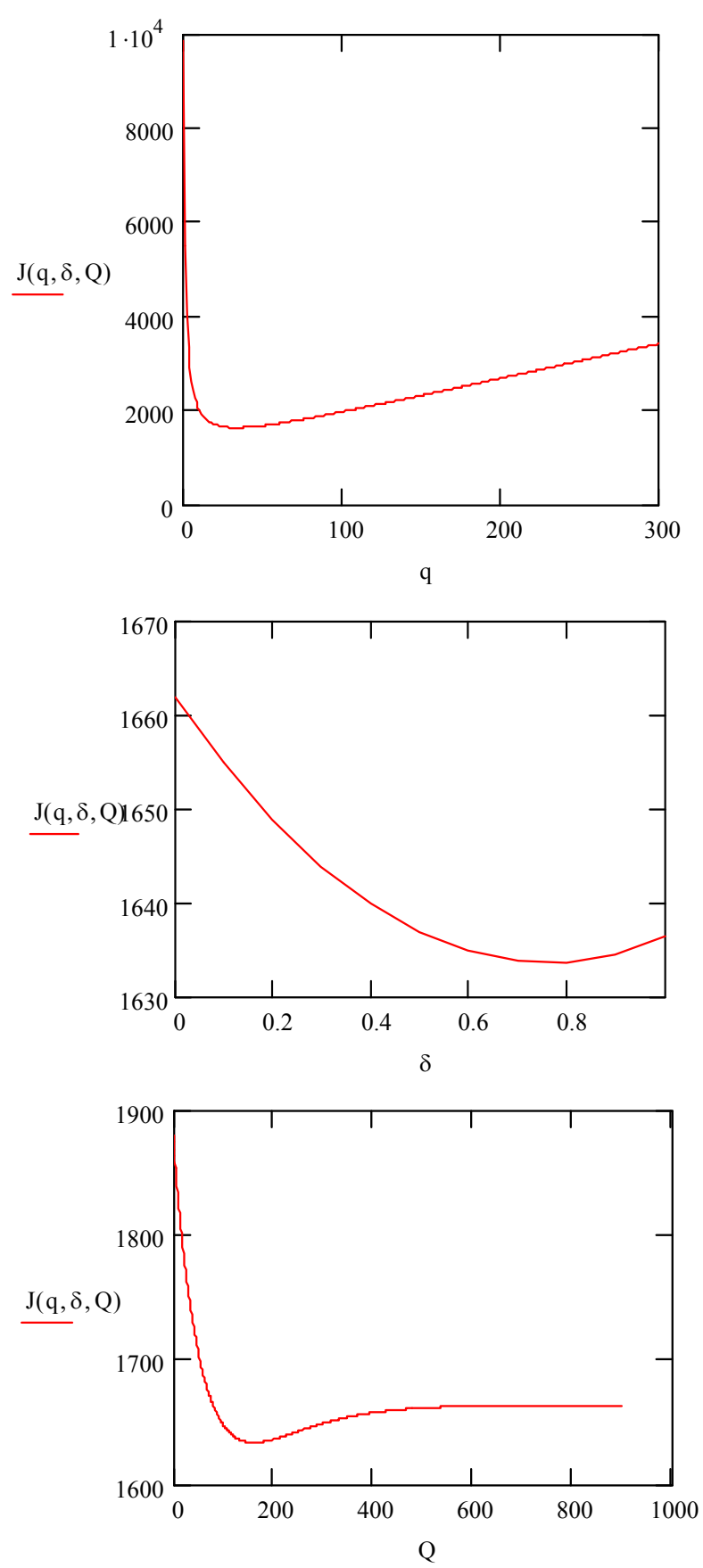
An optimal ordering and disposal policy can be determined by minimising the cost rate function $J(q, M, Q)$ given in (7) with respect to the decision variables $q, M$ and $Q$. Because the Hessian of $J$ evaluated at any $(q, M, Q)$ involves complicated terms and is not easily malleable, it is difficult to establish necessary and sufficient conditions that will guarantee a unique global minimum. This cost function, however, is fairly easy to study numerically. We used the computational software package Mathcad 12 to investigate its behaviour and properties over an extensive set of parameter values. Our numerical trials showed that not only is $J$ a fairly well-behaved function it also has a quasi-convex shape. Figure 2 shows cross sections of the cost function for a given set of parameter values. Note: In order to eliminate the constraint $M \leq Q$ and simplify the optimisation task a bit, we made the change of variable $M=\delta Q$ and substituted this change into the cost function. With this change the variables become $q, \delta$, and $Q$, and the constraint set reduces to $q \geq 0, Q \geq 0$, and $0 \leq \delta \leq 1$. One important characteristic of this function is that the total cost drops sharply as $q, \delta$, and $Q$ are increased, and, akin to general EOQ models, is fairly flat in the area of the minimum total cost.

Table 1 Optimal ordering and disposal policies and costs for various return fractions

\begin{tabular}{llllll}
\hline $1 / \mu$ & $\alpha$ & $q^{*}$ & $M^{*}$ & $Q^{*}$ & $J^{*}$ \\
\hline 20 & 0.1 & 38 & 145 & 183 & $1,682.54$ \\
& 0.3 & 33 & 114 & 152 & $1,470.10$ \\
& 0.5 & 29 & 89 & 124 & $1,312.70$ \\
50 & 0.7 & 24 & 68 & 102 & $1,245.92$ \\
& 0.9 & 20 & 54 & 86 & $1,281.55$ \\
& 0.1 & 38 & 147 & 187 & $1,730.09$ \\
& 0.3 & 34 & 124 & 162 & $1,633.56$ \\
& 0.5 & 30 & 104 & 142 & $1,603.32$ \\
& 0.7 & 27 & 89 & 126 & $1,639.56$ \\
& 0.9 & 24 & 77 & 113 & $1,733.72$ \\
& 0.1 & 38 & 150 & 190 & $1,787.96$ \\
& 0.3 & 35 & 133 & 172 & $1,802.01$ \\
& 0.5 & 32 & 119 & 158 & $1,863.35$ \\
& 0.7 & 30 & 107 & 145 & $1,965.84$ \\
& 0.9 & 27 & 97 & 135 & $2,102.74$ \\
& 0.1 & 39 & 157 & 197 & $1,905.69$ \\
& 0.3 & 38 & 151 & 191 & $2,123.22$ \\
& 0.5 & 37 & 146 & 186 & $2,348.20$ \\
& 0.7 & 36 & 141 & 181 & $2,579.88$ \\
& 0.9 & 35 & 137 & 176 & $2,817.55$ \\
\hline
\end{tabular}

Notes: Parameters: $D=400, \theta=15, \mu$ and $\alpha$ vary, $h=15, K_{1}=30, C_{1}=3, K_{2}=30$, $C_{2}=3$. Also, for each $\mu$, the EOQ's are $38,33,28,22$, and 13 as $\alpha$ is increased. 
Table 2 Optimal cost components for various return fractions

\begin{tabular}{|c|c|c|c|c|}
\hline $1 / \mu$ & $\alpha$ & $J_{l}$ & $J_{2}$ & $J_{3}$ \\
\hline \multirow[t]{5}{*}{20} & 0.1 & 318 & 1,365 & 0 \\
\hline & 0.3 & 377 & 1,092 & 1 \\
\hline & 0.5 & 473 & 826 & 14 \\
\hline & 0.7 & 579 & 597 & 70 \\
\hline & 0.9 & 656 & 433 & 193 \\
\hline \multirow[t]{5}{*}{50} & 0.1 & 359 & 1,368 & 4 \\
\hline & 0.3 & 488 & 1,118 & 27 \\
\hline & 0.5 & 615 & 902 & 86 \\
\hline & 0.7 & 722 & 730 & 188 \\
\hline & 0.9 & 805 & 598 & 331 \\
\hline \multirow[t]{5}{*}{100} & 0.1 & 383 & 1,385 & 20 \\
\hline & 0.3 & 537 & 1,181 & 84 \\
\hline & 0.5 & 671 & 1,010 & 182 \\
\hline & 0.7 & 784 & 871 & 311 \\
\hline & 0.9 & 879 & 758 & 465 \\
\hline \multirow[t]{5}{*}{500} & 0.1 & 369 & 1,455 & 82 \\
\hline & 0.3 & 499 & 1,372 & 252 \\
\hline & 0.5 & 621 & 1,298 & 429 \\
\hline & 0.7 & 736 & 1,230 & 613 \\
\hline & 0.9 & 845 & 1,169 & 803 \\
\hline
\end{tabular}

Notes: Parameters: $D=400, \theta=15, \mu$ and $\alpha$ vary, $h=15, K_{1}=30, C_{1}=3, K_{2}=30$,

$$
C_{2}=3
$$

\subsection{Implications of returns on the optimal procurement and disposal policy}

In this section we present some numerical examples along with interpretations to illustrate the influence that changing the fraction returned $\alpha$ and other parameters have on the optimal ordering and disposal policy. Recall that $\alpha$ is equal to $\lambda / \mu D$ and represents the expected proportion of returned products relative to current demand. In our main illustrative example, we set $D=400, \theta=15, h=15, K_{1}=K_{2}=30$, and $C_{1}=C_{2}=3.1 / \mu$ is given the values $20,50,100$ and 500 to represent small to large expected inflows per return occurrence, respectively. $\alpha$ is varied as $0.10,0.30,0.50,0.70$, and 0.90 to show instances ranging from low to high customer return rates relative to actual demand. Observe that for fixed $D$ and $\mu$, increasing $\alpha$ means that returns are arriving at an increasing frequency, i.e., $\lambda$ is increased. Our numerical results for this data set are summarised in Table 1 and Table 2, where we have used the non-linear solver in Mathcad 12 to optimise our cost function. The values for the optimal solution were rounded to their nearest integer and are given in the original variables $q, M$, and $Q$.

Table 1 shows optimal ordering and disposal quantities and total costs for various return proportions $\alpha$ and expected size per return instance $1 / \mu$, while Table 2 presents the 
individual cost components. $J_{1}$ are the costs of holding inventory or the first term in (7); $J_{2}$ and $J_{3}$ are the ordering and disposal costs or the last two terms in (7), respectively.

Table 3 Optimal ordering and disposal quantities for different parameter values

\begin{tabular}{|c|c|c|c|c|c|c|}
\hline$\theta$ & $1 / \mu$ & $\alpha$ & $q^{*}$ & $M^{*}$ & $Q^{*}$ & $J^{*}$ \\
\hline \multirow[t]{4}{*}{3} & 20 & 0.1 & 38 & 144 & 182 & $1,682.54$ \\
\hline & & 0.5 & 28 & 85 & 116 & $1,317.37$ \\
\hline & 100 & 0.1 & 38 & 148 & 186 & $1,799.25$ \\
\hline & & 0.5 & 31 & 107 & 142 & $1,994.81$ \\
\hline \multirow[t]{4}{*}{15} & 20 & 0.1 & 38 & 145 & 183 & $1,682.54$ \\
\hline & & 0.5 & 29 & 89 & 124 & $1,312.70$ \\
\hline & 100 & 0.1 & 38 & 150 & 190 & $1,787.96$ \\
\hline & & 0.5 & 32 & 119 & 158 & $1,863.35$ \\
\hline \multirow[t]{4}{*}{40} & 20 & 0.1 & 38 & 144 & 184 & $1,682.53$ \\
\hline & & 0.5 & 29 & 91 & 128 & $1,311.13$ \\
\hline & 100 & 0.1 & 38 & 151 & 192 & $1,784.51$ \\
\hline & & 0.5 & 33 & 122 & 162 & $1,832.18$ \\
\hline \multirow[t]{4}{*}{100} & 20 & 0.1 & 38 & 142 & 184 & $1,682.53$ \\
\hline & & 0.5 & 29 & 92 & 130 & $1,310.38$ \\
\hline & 100 & 0.1 & 38 & 152 & 192 & $1,783.03$ \\
\hline & & 0.5 & 32 & 124 & 164 & $1,819.52$ \\
\hline
\end{tabular}

Notes: Parameters: $D=400, \theta, \mu$, and $\alpha$ vary, $h=15, K_{1}=30, C_{1}=3, K_{2}=30$ and $C_{2}=3$

Furthermore to show how disposal opportunities and their expected frequency of occurrence (i.e., parameter $\theta$ ) influence the performance of the system, $\theta$ is given the values $3,15,40$ and 100 ; values representing fairly rare to highly frequent occasions for disposal. A sample of our results is shown in Table 3. Finally, to measure and illustrate the impact of disposal costs on the optimal policy parameters and costs, we varied the cost of disposition from low to high in relation to the cost of ordering. These results are displayed in Table 4.

The results of Tables 1,2,3 and 4 exemplify the typical behaviour of the optimal policy and costs we observed for a wide variety of parameter values. To comment on the pattern of behaviour exhibited in these tables, let us use Heyman's terminology and refer to $q+Q$ as the 'keep level'. Recall that, in our model, a disposal takes place only when the inventory position exceeds this keep level and an opportunity to liquidate or redistribute excess inventory comes along.

First, one important observation gleaned from our computational experiments relates to the form of the optimal policy. Our results show that when a disposal is called for, the optimal policy dictates that enough units are to be disposed of to reduce current inventory levels to well below keep levels. In other words, the optimal $\mathrm{M}$ is an interior-point solution in $[0, Q]$. This was the case in a large number of parameter settings we experimented with. In view of this, we conjecture that the ordering and disposal policy proposed in this paper is indeed the optimal policy for the problem at hand. 
Table 4 Optimal ordering and disposal quantities and costs for varying disposal costs

\begin{tabular}{lllllll}
\hline$\left(K_{2}, C_{2}\right)$ & $1 / \mu$ & $\alpha$ & $q^{*}$ & $M^{*}$ & $Q^{*}$ & $J^{*}$ \\
\hline$(1,0.1)$ & 20 & 0.1 & 38 & 76 & 84 & $1,682.30$ \\
& 0.3 & 34 & 62 & 67 & $1,465.88$ \\
& 0.5 & 29 & 51 & 57 & $1,285.39$ \\
& 50 & 0.1 & 38 & 77 & 85 & $1,722.38$ \\
& 0.3 & 35 & 68 & 75 & $1,590.35$ \\
$(30,3)$ & 0.5 & 32 & 60 & 66 & $1,488.86$ \\
& & 0.1 & 38 & 144 & 182 & $1,682.54$ \\
& & 0.3 & 33 & 114 & 152 & $1,470.10$ \\
& & 0.5 & 29 & 89 & 124 & $1,312.70$ \\
& & 0.1 & 38 & 147 & 187 & $1,730.09$ \\
& 50 & 0.3 & 34 & 124 & 162 & $1,623.56$ \\
& & 0.5 & 30 & 104 & 142 & $1,603.32$ \\
& & 0.1 & 38 & 245 & 345 & $1,682.54$ \\
& 20 & 0.3 & 33 & 224 & 282 & $1,470.56$ \\
& & 0.5 & 28 & 168 & 217 & $1,322.93$ \\
& & 0.1 & 38 & 290 & 347 & $1,732.38$ \\
& 50 & 0.3 & 34 & 236 & 291 & $1,657.79$ \\
& & 0.5 & 29 & 190 & 243 & $1,705.40$ \\
\hline
\end{tabular}

Note: Parameters: $D=400, \theta=15, h=15, \alpha$ and $\mu$ vary, $K_{1}=30, C_{1}=3, K_{2}$ and $C_{2}$ vary

Second, we wanted to find out whether the economic order quantity (EOQ) based on net demand (demand minus expected return) can be used as a quick-and-dirty formula to calculate $q^{*}$. This quantity is given by $E O Q=\sqrt{\frac{2 a D K_{1}}{h_{1}}}$. This formula or a modification of it has been shown to work well in the literature (for example, see Van der Laan and Teunter, 2006). In our case the EOQ also turned out to be a good approximation of $q^{*}$, but only for low return rates and when the expected batch-size per return is small. In general, we found that the EOQ undershoots $q^{*}$ by a fair margin when the frequency of returns is relatively high or when the batch-size of returns $1 / \mu$ is large (see Table 1 for example).

For the data shown in Table 1, Table 3, and Table 4 we see that the optimal policy parameters are all decreasing as $\alpha$ is increased as one would expect. In other words, with increasing return rates, the order frequency is sharply reduced by lowering the optimal order quantity $q^{*}$, which leads to a decrease in ordering costs. Inventory carrying costs, on the other hand, begin to increase rapidly and, to keep them in check, it becomes optimal to lower keep levels and to resort to frequent disposals. As mentioned earlier, $q^{*}$ does not drop as fast as the EOQ prescribes when net demand falls. That is, $q^{*}$ incorporates the possibility of future disposals and is calculated so as to create an overall balance between the costs of holding, ordering, and disposal.

In turn, the optimal total costs may exhibit a different behaviour as $\alpha$ is changed in the specified range. These costs may first decrease and then increase or they may just increase as $\alpha$ is increased. The actual behaviour depends on the arrival frequency of returns and especially on their magnitude. As can be seen from Table 1 and Table 2, for a relatively low expected return batch-size, total costs are decreasing until the return 
frequency reaches a certain threshold before they start increasing. For example, when the expected batch-size per return is $1 / \mu=20$, this critical threshold is as high as $\alpha=70 \%$.

This observation leads to the following remark. Some companies try to prevent returns when it is possible to do so, either by stating a zero-returns policy from the outset or by negotiating special agreements with their suppliers to have them handle all returns. Under short replenishment lead times, the results of Table 1, Table 2, Table 3 and Table 4 tell us the policy of accepting no returns or just a few is not always the best course of action as it depends very much on the nature of the return flow. If returns arrive in smaller bunches, total costs may decrease for a while even as the flow intensity increases. The increase in holding and disposal costs because of returns is more than offset by the savings in ordering costs because of smaller $q^{*}$. However, when the batch-size of returns is sufficiently large, accepting returns can be a costly proposition even if such returns arrive slowly.

Consider now the effect of changes in parameter $\theta$ on the optimal control parameters and costs. Table 3 provides an illustration. In general we found that $q^{*}$ does not appear to be affected very much by whether disposal opportunities are aplenty or scarce. On the other hand, keep and dispose-down-to levels change in a predictable way in response to changes in $\theta$. If opportunities for disposal are rare, we lower keep levels so we can take advantage of them sooner to lower costs when they do occur. If they happen fairly frequently, we set keep levels higher because we will have many chances to lower costs through disposals when it is convenient. Also, note that for a given $\mu$, total costs are decreasing in $\theta$.

In Table 4 we illustrate the effect of disposal costs on the optimal policy and costs. The results agree with intuition. If disposal costs are cheap in comparison to ordering costs, we set keep levels fairly low and dispose of inventory whenever we can. If these same costs are expensive, we set keep levels fairly high with the intent of keeping and re-using most of the returned inventory to satisfy future demand.

Let us now return to the question of refurbishment costs and how they can be incorporated into the objective function (7) when the cost of refurbishing a unit depends on time. For any ordering and disposal policy given by $q, M$, and $Q$, recall that the expected number of orders per unit time is given by $D f_{1}(0)=a D / A(q, M, Q)$, where $a D$ is the net demand and $A(q, M, Q)$ is given in (6). Quantity $A$ in this formula can be interpreted as the order quantity in an equivalent deterministic EOQ model with effective demand $a D$ and an order frequency equal to the one in the stochastic model. Note that $A$ would be equal to $\mathrm{q}$ if returns never occurred (i.e., $\lambda=0$ ). Consequently, the average inventory resulting from replenishments at any point in time can be approximated by $A / 2$. Now $E\left[I P_{0}\right]$ represents the total average inventory, which includes both newly ordered and returned items. We can use the difference $E\left[I P_{0}\right]-A / 2$ to approximate the long-run average returned inventory. Thus, if $C_{3}$ is the refurbishment cost per unit per unit time, the expected total refurbishment costs per unit time can be given by $C_{3}\left(E\left[I P_{0}\right]-A / 2\right)$.

We incorporated this cost component into the objective function (7) to see how the optimal solution is affected by it. We found that the inclusion of this cost does not change our conclusions or the overall behaviour of the optimal policy and costs described above. Naturally the addition of refurbishment costs into (7) will affect the disposal decision in that keep and dispose-down-to levels are lower than the ones with these costs not included. To illustrate, suppose $D=400,1 / \mu=20, \alpha=0.3, \theta=15, h=15, K_{1}=K_{2}=30$, $C_{1}=C_{2}=3$, and $C_{3}=1.5$. In this case, the optimal policy prescribes values of $q^{*}=33$, $M^{*}=105$, and $Q^{*}=140$ and the optimal total costs are $\$ 1,482.66$ (compare these values 
with those given in Table 1). The optimal holding, ordering, disposal, and refurbishment costs are $\$ 376.03, \$ 1,092.52, \$ 1.58$ and $\$ 12.52$, respectively.

An alternative way of incorporating the cost of acquisition, refurbishment, or handling associated with returns into the model is simply to allow this cost to be part of the unit disposal cost. Disposing of refurbished items after they have been restocked is equivalent to foregoing the value that has been added to them and, thus, $C_{2}$ should reflect this fact. If the refurbishment cost is high, however, this will translate into a higher $C_{2}$ and, therefore, into higher keep levels. In effect, this will lead to fewer disposals.

In closing this section, we note that the order point was set equal to 0 for this case of zero lead time. However, it may be desirable to allow for a planned shortage due to the potential of returns. In fact, this may lead to lower total costs if the holding cost is relatively high. This backorder case can be dealt with easily given our previous analysis. Let $I P^{\prime}$ represent the inventory position when $\mathrm{w}$ is the number of backorders that are accumulated when an order of size q is received. Then, $I P^{\prime}=I P_{0}-w, w \geq 0$. If we let $f^{\prime}(x)$ denote the probability density function of $I P^{\prime}$, then $f^{\prime}(x)=f(x+w)$, where $x \geq-w$ and $f($.) is given in (6). Hence, an expression for the total costs (including backorder costs) can be derived quickly from (6).

\subsection{Cost model with non-zero lead time}

In this section we look at the more complicated case of a positive lead time $L$. Since demand in our model occurs continuously at a constant rate of $\mathrm{D}$ units per unit time, it is tempting to conclude that the reorder point $\mathrm{s}$ should be set equal to DL modulo $\mathrm{q}$ so that orders will arrive just as inventory reaches zero. However, as we shall see later, this may not be the best course of action when there is a chance of an inventory increase during a lead time because of returns. Therefore, a 'planned' shortage can be risked by setting the reorder point below the expected demand during lead time. Such a shortage may not materialise because enough returns may arrive in the interim to raise inventory so as to eliminate the risk.

To formulate an exact cost model in this situation, we need to know the stationary distribution of the net inventory $I N(t)$. In principle, the derivation of the distribution of $I N(t)$ can be obtained from the following basic relationship (see Figure 1):

$$
I N(t+L)=I P(t)-D(t, t+L)+R(t, t+L)-S(t, t+L)
$$

Expression (9) holds since lead time is assumed to be constant and, thus, crossing of orders cannot happen. The stationary distribution of $I P(t)$ can be obtained easily from our previous analysis. Let $I P$ represent the steady-state inventory position for the non-zero lead time case. $I P$ can be given as $I P=I P_{0}+s$, where $\mathrm{s}$ is the reorder point. Thus, $I P$ is just a translation of $I P_{0}$. The stationary distribution of $I P_{0}$ has already been derived in Section 4.1. Furthermore, in our case, $D(t, t+L)$ is equal to DL for all $t$. As noted previously, $R(t)$ is a compound Poisson process, which has independent and stationary increments. Thus, we have $R(t, t+L)=R(L)=\sum_{n=1}^{N(L)} R_{n}$ for all t. Moreover, the times at which inventory disposals occur are events of a Poisson process $\bar{N}(t)$ with rate $\theta \int_{q+Q}^{+\infty} f_{4}(x) d x$. The amount disposed of at the nth occurrence of this process is 
$S_{n}=I P_{n}-(s+q+M) . I P_{n}$ is the inventory position at the epoch of the nth event of $\bar{N}$. Clearly, $S(t, t+L)=S(L)=\sum_{n=1}^{\bar{N}(L)} S_{n}$ for all $\mathrm{t}$, since the amounts disposed of in disjoint intervals are independent. Letting $t \rightarrow \infty$, let us rewrite equation (9) as:

$$
I N=s-D L+I P_{0}+R(L)-S(L)
$$

where $I N$ is the steady-state net inventory for the positive lead-time case.

Although the stationary distribution of each term on the right hand-side of (10) is readily available, obtaining the exact distribution of $I N$ from (10) is no easy task. The random variables involved in (10) are not independent of each other. Consequently, rather than try to develop the exact distribution for $I N$, we will resort to an approximation.

One important approximation that has worked well in practice and has often been used in the literature is the normal distribution (see Muckstadt and Isaac, 1981; Van der Laan et al., 1996a, 1996b; Zipkin, 2000). That is, the probability density function of $I N$ is approximated by a normal density. We will use equation (10) to approximate both the mean $v$ and variance $\sigma^{2}$ of this normal distribution. That is, $v$ and $\sigma^{2}$ are calculated as follows:

$$
\begin{aligned}
v & =E[I N]=s-D L+E\left[I P_{0}\right]+E[R(L)]-E[S(L)] \\
\sigma^{2} & =V A R[I N] \approx V A R\left[I P_{0}\right]+\operatorname{VAR}[R(L)]+\operatorname{VAR}[S(L)]
\end{aligned}
$$

In the calculation of $\sigma^{2}$ the covariance terms were left out. Now $\operatorname{VAR}\left[I P_{0}\right]=E\left[I P_{0}^{2}\right]-E\left[I P_{0}\right]^{2}$, where $E\left[I P_{0}\right]$ and $E\left[I P_{0}^{2}\right]$ can both be derived from (6). Moreover, $R(L)$ is compound Poisson random variable; thus, $E[R(L)]=\lambda E[R] L$ and $\operatorname{VAR}[R(L)]=\lambda E\left[R^{2}\right] L . E[S(L)]$ and $\operatorname{VAR}[S(L)]$ are given by

$$
\begin{gathered}
E[S(L)]=\left(\theta \int_{q+Q}^{+\infty} f_{4}(x) d x\right) E[S] L \\
V A R[S(L)]=\left(\theta \int_{q+Q}^{+\infty} f_{4}(x) d x\right) E\left[S^{2}\right] L
\end{gathered}
$$

where $S$ is the (random) amount withdrawn from inventory at a disposal epoch. Note that $\mathrm{S}$ can also be given as $S=X+Q-M$, where $X$ is the amount in excess of $s+q+Q$ at a disposal epoch. The distribution of $X$ is given by

$$
\begin{aligned}
\Psi(x) & =P[X \leq x]=P\left[I P_{0} \leq q+Q+x / I P_{0}>q+Q\right] \\
& =\frac{F(q+Q+x)-F(q+Q)}{1-F(q+Q)}, x \geq 0
\end{aligned}
$$

where $F$ is the cumulative distribution of $I P_{0}$.

When the distribution for the amount returned is given by $\bar{G}(x)=e^{-\mu x}, x \geq 0$, expressions for all quantities entering into the calculation of $v$ and $\sigma$ given in (11) as functions of the decision variables $s, q, M$ and $Q$ are given in the appendix. 
In addition, we performed a simulation study and diagnostic checks to verify the accuracy of the normal distribution as a substitute to the distribution of net inventory $I N$ given in (10). Based on our test trials, this approximation turned out to be fairly good and represented a reasonable model for the distribution of net inventory. Minor departures from the normal distribution occurred mostly along the upper-part in the right-tail of the distribution.

We are now ready to give an approximate cost rate function for this case of non-zero lead time. Let $u(x)$ represent the density function of $I N . u(x)$ is approximated by a normal density with mean $v$ and variance $\sigma^{2}$ given in (11). Let $I$ and $B$ represent the long-run on-hand inventory and number of backorders, respectively. Then,

$$
\begin{aligned}
& E[B]=\int_{-\infty}^{0} u(x) d x=\sigma \phi\left(\frac{v}{\sigma}\right)-v \Phi\left(-\frac{v}{\sigma}\right) \\
& E[I]=E[I N]+E[B]=v+\sigma \phi\left(\frac{v}{\sigma}\right)-v \Phi\left(-\frac{v}{\sigma}\right)
\end{aligned}
$$

$\phi($.$) and \Phi($.$) are the standard normal density and standard normal distribution functions,$ respectively. Since orders and disposals are based on the inventory position, the expected ordering costs and the expected disposal costs per unit time are the same as those obtained for the zero-lead-time case. Therefore, if we let $\mathrm{h}$ and $\mathrm{b}$ denote the holding and backorder cost, respectively, per unit per unit time, the cost rate function can be given by

$$
\begin{aligned}
J(s, q, M, Q) & =h v+(h+b)\left(\sigma \phi\left(\frac{v}{\sigma}\right)-v \Phi\left(-\frac{v}{\sigma}\right)\right)+\frac{a D\left(K_{1}+C_{1} q\right)}{A} \\
& +\frac{\theta a(r+1)}{\mu r \bar{A}}\left[C_{2}\left(\frac{1}{\mu r}+M-Q\right)-K_{2}\right]
\end{aligned}
$$

The goal is to determine values for $s, q, M$, and $Q$ that minimise the cost function given in (13). First, for fixed $q, M$, and $Q$, it can be checked that $J$ is convex in s. Therefore, the optimal value of $\mathrm{s}$ is the unique solution to $\partial J / \partial s=0$. It follows that $s^{*}$ satisfies the following equation: $\Phi(-v / \sigma)=h /(b+h)$. Thus, the optimal value of $\mathrm{s}$ is then given by

$$
s^{*}=D L+E[S(L)]-\left(E\left[I P_{0}\right]+E[R(L)]+\sigma \Phi^{-1}(h /(b+h))\right)
$$

The optimisation problem can be reduced to a search in the three variables $q, M$, and $Q$ by substituting this expression for $s^{*}$ into the objective function (13).

To provide some numerical results for the lead-time case, we again used the non-linear solver in Mathcad 12 to perform the optimisation task. Furthermore, to compare the results of this case to the ones discussed without lead times, we shall use the same example which we presented in Section 4.2. That is, we let $D=400, \theta=15, h=15$, and $b=20, K_{1}=K_{2}=30$, and $C_{1}=C_{2}=3$. The lead time $L$ is given the values 1,6 and 12. To highlight the effects of the Compound Poisson nature of the return flow, we let $1 / \mu$ take on the values 20 and 100 , while $\alpha$ is varied as $0.1,0.3,0.5,0.7$ and 0.9 . Table 5 displays our results. 
Table 5 Optimal policy values and costs for various parameters and lead times

\begin{tabular}{|c|c|c|c|c|c|c|c|}
\hline$L$ & $1 / \mu$ & $\alpha$ & $s^{*}$ & $q^{*}$ & $M^{*}$ & $Q^{*}$ & $J^{*}$ \\
\hline \multirow[t]{10}{*}{1} & 20 & 0.1 & 328 & 76 & 148 & 152 & $1,862.83$ \\
\hline & & 0.3 & 244 & 82 & 156 & 162 & $1,986.36$ \\
\hline & & 0.5 & 159 & 81 & 153 & 160 & $2,026.70$ \\
\hline & & 0.7 & 69 & 75 & 150 & 157 & $2,075.96$ \\
\hline & & 0.9 & -21 & 68 & 151 & 159 & $2,218.54$ \\
\hline & 100 & 0.1 & 321 & 100 & 291 & 294 & $2,615.65$ \\
\hline & & 0.3 & 229 & 112 & 309 & 313 & $3,401.74$ \\
\hline & & 0.5 & 136 & 114 & 319 & 324 & $3,961.75$ \\
\hline & & 0.7 & 43 & 113 & 329 & 334 & $4,475.95$ \\
\hline & & 0.9 & -48 & 111 & 340 & 346 & $5,006.02$ \\
\hline \multirow[t]{10}{*}{6} & 20 & 0.1 & 2,127 & 99 & 316 & 316 & $2,597.58$ \\
\hline & & 0.3 & 1,648 & 108 & 318 & 318 & $3,304.82$ \\
\hline & & 0.5 & 1,168 & 105 & 297 & 300 & $3,732.76$ \\
\hline & & 0.7 & 679 & 95 & 272 & 275 & $4,078.38$ \\
\hline & & 0.9 & 209 & 81 & 258 & 261 & $4,515.71$ \\
\hline & 100 & 0.1 & 2,126 & 129 & 667 & 668 & $4,287.86$ \\
\hline & & 0.3 & 1,641 & 143 & 651 & 652 & $6,320.03$ \\
\hline & & 0.5 & 1,150 & 142 & 630 & 632 & $7,745.25$ \\
\hline & & 0.7 & 671 & 136 & 617 & 619 & $9,020.08$ \\
\hline & & 0.9 & 242 & 129 & 619 & 621 & $10,324.48$ \\
\hline \multirow[t]{10}{*}{12} & 20 & 0.1 & 4,288 & 110 & 381 & 381 & $3,135.72$ \\
\hline & & 0.3 & 3,335 & 121 & 451 & 451 & $4,253.18$ \\
\hline & & 0.5 & 2,377 & 118 & 398 & 398 & $4,959.59$ \\
\hline & & 0.7 & 1,410 & 106 & 353 & 355 & $5,521.62$ \\
\hline & & 0.9 & 471 & 87 & 324 & 326 & $6,155.25$ \\
\hline & 100 & 0.1 & 4,294 & 144 & 934 & 934 & $5,501.06$ \\
\hline & & 0.3 & 3,338 & 159 & 890 & 891 & $8,423.04$ \\
\hline & & 0.5 & 2,366 & 157 & 839 & 840 & $10,450.96$ \\
\hline & & 0.7 & 1,410 & 147 & 800 & 802 & $12,236.19$ \\
\hline & & 0.9 & 563 & 136 & 789 & 790 & $14,066.52$ \\
\hline
\end{tabular}

Notes: Parameters: $D=400, \theta=15, \mu, \alpha$, and $L$ vary, $h=15, b=20, K_{1}=K_{2}=30$, and

$$
C_{1}=C_{2}=3
$$

Our sensitivity analysis and interpretations will be limited to the effect of changes in the fraction returned relative to demand combined with changes in lead times. Comparing the results of Table 5 to those of Table 1, Table 2, Table 3, and Table 4, we see that when lead times are considered, the behaviour of the optimal policy parameters and total costs may take a different look. First, as we remarked earlier, to account for the potential of returns that can drive serviceable inventory to well above the reorder point during a replenishment lead time, an order need not be placed when the inventory position is equal to DL. In fact, the reorder point $s^{*}$ should be set much lower than DL as evidenced in Table 5. In other words, as long as demand-rate fluctuations remain low over time, 
shortages are intentionally planned as their full impact may not be felt because of the possibility of an inventory increase during a lead time.

In addition, for fixed $L$, we also see that the reorder point $s^{*}$ drops substantially as the fraction returned increases, while the order quantity $q^{*}$ is only moderately adjusted at times first up and then down (see Table 5). Thus, even though expected net demand is dropping, it is sometimes better to raise $q^{*}$ for a while. The larger $q^{*}$ together with returns adding to inventory will delay ordering and, thus, reduce ordering costs. The reduction plus the occasional disposal will temporarily be enough to absorb the increase in holding and backorder costs. We remark that, unlike the zero lead-time case discussed in Section 4.2, the EOQ based on net demand does not provide a simple approximation to $q^{*}$ in this case. $q^{*}$ is set much higher than what the EOQ would suggest.

Changes in $Q^{*}$ or the difference between order-up-to levels and keep levels are far more complicated. They appear to depend on the nature of the return flow one experiences and also on lead time length. As can be seen from Table 5, for a given $L, Q^{*}$ displays different behaviours as $\alpha$ is increased. For shorter lead times and smaller returned lots, $Q^{*}$ can first increase and then decrease as $\alpha$ changes. In other instances, a different response is observed. Thus, to achieve that fine balance in the costs involved, the optimal policy can take many forms indeed.

Another key difference is seen in the behaviour of $Q^{*}-M^{*}$ or the gap between keep levels and dispose-down-to levels. Compared to the results of Table 1 this gap gets narrower and, for a sufficiently long lead time, it drops to 0 . Thus, in this instance, disposals are limited only to the excess that has accumulated over and above current keep levels.

In the no lead-time case we saw that total costs may decrease for a while and then increase as return rates increase, i.e., accepting some returns may temporarily result in a cost advantage. However, as shown in Table 5, this is not the case for a non-zero lead time under the same demand, return and cost conditions. Here total costs show a steady increase in the relevant $\alpha$ range. The increase is more pronounced for longer lead times.

Finally, all other things being equal the qualitative effects of varying lead times on $s^{*}$, $q^{*}, M^{*}, Q^{*}$ and overall costs are as one would expect. For fixed $\mu$ and $\alpha$, an increase in lead time results in an increase in the values of the policy parameters and the total cost of replenishment, carrying inventory, shortage, and disposal. While we believe the qualitative effects described in Table 5 to be typical, nothing general can be established analytically due in large part to the complicated form of the cost function given in (13).

\section{Summary and conclusions}

In this paper we considered a replenishment control system that incorporates random returns and disposals of excess inventory when necessary. In this model we assumed that the demand rate is constant and deterministic, and that the total amount of returns accumulated over a period of time is represented by a compound Poisson process. Moreover we assumed that inventory reductions occur only when beneficial opportunities for disposal or relocation arise at times governed by a Poisson process.

We zeroed in on serviceable inventory and its day-to-day fluctuations. We used an $(s, q)$ inventory policy to control the when and how much to order when the replenishment lead-time is constant. In this case, the target for the inventory position is $s+q$. This target level may occasionally be exceeded because too many products have 
been brought back and added to inventory. When an opportunity for a disposal arises, it is pursued only when the inventory position is above target $s+q+Q$. Each disposal restores inventory levels to target $s+q+M$, where $0 \leq M \leq Q$.

We derived expressions for the total costs per unit time, which consisted of ordering, holding, shortage, and disposal costs. This model makes specific the influence that returns arriving in varying quantities have on the behaviour of optimal procurement and disposal policy and costs. Our sensitivity analysis showed that this behaviour can take unexpected forms. The actual behaviour depends on the interplay of lead times, the arrival frequency of returns, and especially on the size of the amount returned, among other things. For example, in the presence of lead times, the order quantity $q^{*}$ may go up for a while as expected net demand drops before it is lowered again. Also, even in the case of short lead times, we observed that the EOQ formula based on net demand (demand minus expected return) is not always a good substitute for calculating $q^{*}$. This approximation works well only when return rates are low and/or the expected batch-size per return is small.

Another important observation taken from our computational results pertains to the optimal amount that should be disposed of as a result of excessive inventory due to many returns. In general, depending on lead time length, a sufficient amount is cleared so that the resultant inventory levels are well below current keep levels. When lead times are long, however, it is only necessary to clear the current excess over keep levels.

Similarly, some companies try to circumvent the hassle of returns by simply stating a zero-returns policy or by striking special arrangements with their customers to take sole responsibility of the returns. Our numerical examples show that this strategy of total avoidance is not always the best as it depends on the nature of the return flow. When lead times can be neglected, accepting and restocking returns can temporarily lead to lower total costs (see Table 1, Table 2, Table 3, and Table 4 for example).

Finally, since the demand rate is constant over time in our model it may be tempting to claim that the reorder point should be set equal to the demand during lead time so that orders will arrive just as the inventory hits zero. This is not the optimal decision in the presence of returns when there is a chance of inventory increases during a lead time. In fact a shortage risk is taken by setting the reorder point well below the demand during lead time. Such a shortage may not happen at all if enough items are returned during a lead time. Our model allows to quantify how much of a shortage can be risked given the actual demand, the frequency and size of returns, and the system's costs.

In terms of future work, there are some worthy extensions to the model presented in this paper. Our model was intended for products that have low-demand uncertainty and long life cycle, e.g., a functional product. It would be worthwhile to extend this analysis to products that have demand uncertainty that cannot be ignored. One possibility would be to represent demand uncertainty by a compound Poisson process. Additionally, our analysis has centred on a pragmatic form of stocking and disposal policy specified by four control values, which, we suspect, constitutes an optimal policy for the model analysed in this paper. Future research should attempt to establish its optimality in a formal setting. 


\section{Acknowledgements}

The author wishes to thank two reviewers for their valuable comments and suggestions that have led to a much improved paper.

\section{References}

Azoury, K. and Brill, P.H. (1986) 'An application of the system-point method to inventory models under continuous review', Journal of Applied Probability, Vol. 23, No. 3, pp.778-789.

Azoury, K. and Brill, P.H. (1992) 'Analysis of net inventory in continuous review models with random lead time', European Journal of Operational Research, Vol. 59, No. 3, pp.383-392.

Beltrán, J.L. and Krass, D. (2002) 'Dynamic lot sizing with returning items and disposals', IIE Transactions, Vol. 34, No. 5, pp.437-448.

Blackburn, J.D., Guide, V.D.R.J., Souza, G.C. and Van Wassenhove, L.N. (2004) 'Reverse supply chains for commercial returns', California Management Review, Vol. 46, No. 2, pp.6-22.

Brill, P.H. (2008) Level Crossing Methods in Stochastic Models, Springer Science+Business Media, LLC, New York.

Brill, P.H. and Chaouch, A.B. (1995) 'An EOQ model with random variations in demand', Management Science, Vol. 41, No. 5, pp.927-936.

Brill, P.H. and Posner, M.J.M. (1977) 'Level crossings in point processes applied to queues: single-server case', Operations Research, Vol. 25, No. 4, pp.662-674.

Brill, P.H. and Posner, M.J.M. (1981) 'The system-point method in exponential queues: a level crossing approach', Mathematics of Operations Research, Vol. 6, No. 1, pp.31-49.

Chaouch, A.B. (2001) 'Stock levels and delivery rates in vendor-managed inventory programs', Production and Operations Management, Vol. 10, No. 1, pp.31-44.

Chaouch, A.B. (2007) 'Inventory control and periodic price-discounting campaigns', Naval Research Logistics, Vol. 54, No. 1, pp.94-108.

Cohen, M.A., Nahmias, S. and Pierskalla, W.P. (1980) 'A dynamic inventory system with recycling', Naval Research Logistics Quarterly, Vol. 27, No. 2, pp.289-296.

DeCroix, G.A. (2006) 'Optimal policy for a multiechelon inventory system with remanufacturing', Operations Research, Vol. 54, No. 3, pp.532-543.

DeCroix, G.A. and Zipkin, P.H. (2005) 'Inventory management for an assembly system with product or component returns', Management Science, Vol. 51, No. 8, pp.1250-1265.

DeCroix, G.A., Song, J.S. and Zipkin, P.H. (2005) 'A series system with returns: stationary analysis', Operations Research, Vol. 53, No. 2, pp.350-362.

Fisher, M. (1997) 'What is the right supply chain for your product?', Harvard Business Review, Vol. 75, No. 2, pp.83-93.

Fleischmann, M. and Kuik, R. (2003) 'On optimal inventory control with independent stochastic item returns', European Journal of Operational Research, Vol. 151, No. 1, pp.25-37.

Fleischmann, M., Bloemhof-Ruwaard, J.M., Dekker, R., Van der Laan, E., Van Nunen, J.A.E.E. and Van Wassenhove, L.N. (1997) 'Quantitative models for reverse logistics: a review', European Journal of Operational Research, Vol. 103, No. 1, pp.1-17.

Fleischmann, M., Kuik, R. and Dekker, R. (2002) 'Controlling inventories with stochastic item returns: a basic model', European Journal of Operational Research, Vol. 138, No. 1, pp.63-75.

Heyman, D.P. (1977) 'Optimal disposal policies for a single-item inventory system with returns', Naval Research Logistics Quarterly, Vol. 24, No. 3, pp.385-405.

Inderfurth, K. (1997) 'Simple optimal replenishment and disposal policies for a product recovery system with leadtimes', OR Spectrum, Vol. 19, No. 2, pp.111-122. 
Karaer, Ö. and Lee, H.L. (2007) 'Managing the reverse channel with RFID-enabled negative demand information', Production \& Operations Management, Vol. 16, No. 5, pp.625-645.

Kelle, P. and Silver, E.A. (1989) 'Purchasing policy of new containers considering the random returns of previously issued containers', IIE Transactions, Vol. 21, No. 4, pp.349-354.

Kiesmuller, G.P. and Van der Laan, E.A. (2001) 'An inventory model with dependent product demands and returns', International Journal Production Economics, Vol. 72, No. 1, pp.73-87.

Lonn, S. and Stuart, J.A. (2003) 'Increasing service through aggressive dealer inventory return policies', International Journal of Physical Distribution \& Logistics Management, Vol. 33, No. 6, pp.519-530.

Mahadevan, B., Pyke, D.F. and Fleischmann, M. (2003) 'Periodic review, push inventory policies for remanufacturing', European Journal of Operational Research, Vol. 151, No. 3, pp.536-551.

Muckstadt, J.A. and Isaac, M.H. (1981) 'An analysis of single item inventory systems with returns', Naval Research Logistics Quarterly, Vol. 28, No. 2, pp.237-254.

Richter, K. (1996) 'The EOQ repair and waste disposal model with variable setup numbers', European Journal of Operational Research, Vol. 95, No. 2, pp.313-324.

Schrady, D.A. (1967) 'A deterministic inventory model for repairable items', Naval Research Logistics Quarterly, Vol. 14, No. 3, pp.391-398.

Silver, E.A., Pyke, D.F. and Peterson, R. (1998) Inventory Management and Production Planning and Scheduling, John Wiley \& Sons, New York.

Simpson, V.P. (1978) 'Optimal solution structure for a repairable inventory problem', Operations Research, Vol. 26, No. 2, pp.270-281.

Stock, J., Speh, T. and Shear, H. (2002) 'Many happy (product) returns', Harvard Business Review, Vol. 80, No. 7, pp.16-17.

Teunter, R.H. (2001) 'Economic ordering quantities for remanufacturable item inventory systems', Naval Research Logistics, Vol. 48, No. 6, pp.484-495.

Teunter, R.H. and Van der Laan, E.A. (2002) 'On the non-optimality of the average cost approach for inventory models with remanufacturing', International Journal of Production Economics, Vol. 79, No. 1, pp.67-73.

Van der Laan, E., Dekker, R. and Salomon, M. (1996a) 'Product remanufacturing and disposal: a numerical comparison of alternative control strategies', International Journal Production Economics, Vol. 45, Nos. 1-3, pp.489-498.

Van der Laan, E., Dekker, R., Salomon, M. and Ridder, M. (1996b) 'An (s, Q) inventory model with remanufacturing and disposal', International Journal Production Economics, Vols. 46-47, pp.339-350.

Van der Laan, E., Salomon, M., Dekker, R. and Van Wassenhove, L.N. (1999) 'Inventory control in hybrid systems with remanufacturing', Management Science, Vol. 45, No. 5, pp.733-747.

Van der Laan, E.A. and Teunter, R.H. (2006) 'Simple heuristics for push and pull remanufacturing policies', European Journal of Operations Research, Vol. 175, No. 2, pp.1084-1102.

Yuan, X.M. and Cheung, K.L. (1998) 'Modeling returns of merchandise in an inventory system', OR Spektrum, Vol. 20, No. 3, pp.147-154.

Zipkin, P.H. (2000) Foundations of Inventory Management, McGraw-Hill, New York. 


\section{Appendix}

Assume that the distribution for the amount returned is given by $\bar{G}(x)=e^{-\mu x}, x \geq 0$, and define

$$
\begin{aligned}
& B_{1}(q, M, Q)=\frac{\frac{q^{3}}{3}+b_{1} q\left(1+\frac{\beta q}{2}\right)}{A} \\
& B_{2}(q, M, Q)=\frac{b_{2}\left(1-e^{-\beta q}\right)\left[1+\beta(q+M)+\frac{[\beta(q+M)]^{2}}{2}\right] e^{-\beta M}}{A} \\
& \left.B_{3}(q, M, Q)=\frac{b_{3}\left[(q+Q)^{3}-(q+M)^{3}\right]}{\bar{A}}\right] \\
& \left.B_{4}(q, M, Q)=\frac{\left[1+\beta(q+Q)+\frac{[\beta(q+Q)]^{2}}{2}\right.}{b_{4}\left[-\left\{1+\beta(q+M)+\frac{[\beta(q+M)]^{2}}{2}\right\} e^{\beta(Q-M)}\right.}\right] \\
& \left.B_{5}(q, M, Q)=\frac{\left[1-b_{5}(q+Q)+\frac{\left[b_{5}(q+Q)\right]^{2}}{2}\right]}{\bar{A}}\right]
\end{aligned}
$$

where

$$
\begin{gathered}
\beta=a \mu, b_{1}=2 \alpha / \beta^{2}, b_{2}=2 \alpha / \beta^{3}, b_{3}=(r+a) / 3, b_{4}=2 \alpha r / \beta^{3}, \\
b_{5}=r \mu, b_{6}=2 a(r+1) / b_{5}^{3},
\end{gathered}
$$

$\mathrm{A}$ and $\overline{\mathrm{A}}$ are defined in (6).

Then, $E\left[I P_{0}^{2}\right]=B_{1}-B_{2}+B_{3}+B_{4}-B_{5}, V A R\left[I P_{0}\right]=E\left[I P_{0}^{2}\right]-E\left[I P_{0}\right]^{2}$, and $E\left[I P_{0}\right]$ is given in (8). Also, $E[R(L)]=(\lambda / \mu) L$ and $V A R[R(L)]=2\left(\lambda / \mu^{2}\right) \mathrm{L}$. Using (6) and (12), it can be shown that $\Psi(x)=1-e^{r \mu x}, x \geq 0$. Thus, the random variable $X$ is exponentially distributed with parameter $-r \mu>0$. It follows that $E[S]=E[X]+Q-M=Q-M-1 / r \mu$ and $E\left[S^{2}\right]=V A R[X]+E[S]^{2}=1 /(r \mu)^{2}+(Q-M-1 / r \mu)^{2}$. Using (6) again, we have

$$
\begin{aligned}
& E[S(L)]=-\frac{\theta a(r+1)(Q-M-1 / r \mu) L}{r \mu \bar{A}} \\
& \operatorname{VAR}[S(L)]=-\frac{\theta a(r+1)\left[1 /(r \mu)^{2}+(Q-M-1 / r \mu)^{2}\right] L}{r \mu \bar{A}} .
\end{aligned}
$$

Finally, using all the above, we can express $v$ and $\sigma^{2}$ in (11) as functions of the decision variables $s, q, M$, and $Q$. 